PROCEEDINGS OF THE

AMERICAN MATHEMATICAL SOCIETY

Volume 137, Number 11, November 2009, Pages 3749-3755

S 0002-9939(09)09923-7

Article electronically published on May 21, 2009

\title{
FREE ARAKI-WOODS FACTORS AND CONNES' BICENTRALIZER PROBLEM
}

\author{
CYRIL HOUDAYER
}

(Communicated by Marius Junge)

\begin{abstract}
We show that for any type $\mathrm{III}_{1}$ free Araki-Woods factor $\mathcal{M}=$ $\Gamma\left(H_{\mathbf{R}}, U_{t}\right)^{\prime \prime}$, the bicentralizer of the free quasi-free state $\varphi_{U}$ is trivial. Using Haagerup's Theorem, it follows that there always exists a faithful normal state $\psi$ on $\mathcal{M}$ such that $\left(\mathcal{M}^{\psi}\right)^{\prime} \cap \mathcal{M}=\mathbf{C}$.
\end{abstract}

\section{INTRODUCTION}

Let $\mathcal{M}$ be a separable type $\mathrm{III}_{1}$ factor and let $\varphi$ be a faithful normal (f.n.) state on $\mathcal{M}$. For any $x, y \in \mathcal{M}$, set $[x, y]=x y-y x$ and $[x, \varphi]=x \varphi-\varphi x$. The asymptotic centralizer of $\varphi$ is defined by

$$
\operatorname{AC}(\varphi):=\left\{\left(x_{n}\right) \in \ell^{\infty}(\mathbf{N}, \mathcal{M}):\left\|\left[x_{n}, \varphi\right]\right\| \rightarrow 0\right\} .
$$

Note that $\operatorname{AC}(\varphi)$ is a unital $\mathrm{C}^{*}$-subalgebra of $\ell^{\infty}(\mathbf{N}, \mathcal{M})$. The bicentralizer of $\varphi$ is defined by

$$
\mathrm{AB}(\varphi):=\left\{a \in \mathcal{M}:\left[a, x_{n}\right] \rightarrow 0 \text { ultrastrongly, } \forall\left(x_{n}\right) \in \mathrm{AC}(\varphi)\right\} .
$$

It is well-known that $\operatorname{AB}(\varphi)$ is a von Neumann subalgebra of $\mathcal{M}$, globally invariant under the modular group $\left(\sigma_{t}^{\varphi}\right)$. Moreover, $\operatorname{AB}(\varphi) \subset\left(\mathcal{M}^{\varphi}\right)^{\prime} \cap \mathcal{M}$. If $\operatorname{AB}(\varphi)=\mathbf{C}$, it follows from the Connes-Størmer Transitivity Theorem $([5])$ that $\operatorname{AB}(\psi)=\mathbf{C}$ for any faithful normal state $\psi$ on $\mathcal{M}$. We shall say in this case that $\mathcal{M}$ has trivial bicentralizer. Connes conjectured that any separable type $\mathrm{III}_{1}$ factor should have trivial bicentralizer. If there exists a faithful normal state $\varphi$ on $\mathcal{M}$ such that $\left(\mathcal{M}^{\varphi}\right)^{\prime} \cap \mathcal{M}=\mathbf{C}$, then $\mathcal{M}$ has trivial bicentralizer. Haagerup proved in 7$]$ that the converse holds true. Haagerup's Theorem leads to the uniqueness of the amenable type $\mathrm{III}_{1}$ factor (see [4]). The following type $\mathrm{III}_{1}$ factors are known to have trivial bicentralizer:

(1) The unique amenable $\mathrm{III}_{1}$ factor (Haagerup, [7).

(2) Full factors that have almost periodic states (Connes, 3]).

(3) Free products $\left(\mathcal{M}_{1}, \varphi_{1}\right) *\left(\mathcal{M}_{2}, \varphi_{2}\right)$ such that the centralizers $\mathcal{M}_{i}^{\varphi_{i}}$ have enough unitaries (Barnett, [1]).

In this paper, we show that the bicentralizer is trivial for a large class of type $\mathrm{III}_{1}$ factors, namely the free Araki-Woods factors of Shlyakhtenko ([14). We briefly recall the construction here; see Section 2 for more details. To each real separable

Received by the editors October 7, 2008, and, in revised form, February 16, 2009.

2000 Mathematics Subject Classification. Primary 46L10, 46L54.

Key words and phrases. Free Araki-Woods factors, Connes' bicentralizer problem.

(C)2009 American Mathematical Society 
Hilbert space $H_{\mathbf{R}}$ together with an orthogonal representation $\left(U_{t}\right)$ of $\mathbf{R}$ on $H_{\mathbf{R}}$, one can associate a von Neumann algebra denoted by $\Gamma\left(H_{\mathbf{R}}, U_{t}\right)^{\prime \prime}$, called the free Araki-Woods von Neumann algebra. This is the free analog of the factors coming from the CAR relations. The von Neumann algebra $\Gamma\left(H_{\mathbf{R}}, U_{t}\right)^{\prime \prime}$ comes equipped with a unique free quasi-free state denoted by $\varphi_{U}$, which is always normal and faithful on $\Gamma\left(H_{\mathbf{R}}, U_{t}\right)^{\prime \prime}$. If $\operatorname{dim} H_{\mathbf{R}} \geq 2$, then $\Gamma\left(H_{\mathbf{R}}, U_{t}\right)^{\prime \prime}$ is a full factor. It is of type $\mathrm{III}_{1}$ when $\left(U_{t}\right)$ is non-periodic and non-trivial. If the representation $\left(U_{t}\right)$ is almost periodic, then $\varphi_{U}$ is an almost periodic state and it follows from [14 that the relative commutant of the centralizer of the free quasi-free state is trivial, i.e. if $\mathcal{M}:=\Gamma\left(H_{\mathbf{R}}, U_{t}\right)^{\prime \prime}$, then $\left(\mathcal{M}^{\varphi_{U}}\right)^{\prime} \cap \mathcal{M}=\mathbf{C}$. In the almost periodic case, results in [6] yield $\mathcal{M}^{\varphi_{U}} \simeq L\left(\mathbf{F}_{\infty}\right)$.

When the representation $\left(U_{t}\right)$ has no eigenvectors (e.g. $U_{t}=\lambda_{t}$, the left regular representation of $\mathbf{R}$ on $\left.L^{2}(\mathbf{R}, \mathbf{R})\right)$, then the centralizer $\mathcal{M}^{\varphi_{U}}$ is trivial. It was unknown in general whether or not $\Gamma\left(H_{\mathbf{R}}, U_{t}\right)^{\prime \prime}$ has trivial bicentralizer. Even though the centralizer of the free quasi-free state $\varphi_{U}$ may be trivial, we will show that the bicentralizer of $\varphi_{U}$ is always trivial. The main result of this paper is the following:

Theorem. Let $\mathcal{M}:=\Gamma\left(H_{\mathbf{R}}, U_{t}\right)^{\prime \prime}$ be a free Araki-Woods factor of type $\mathrm{III}_{1}$. Denote by $\varphi_{U}$ the free quasi-free state. Then $\mathrm{AB}\left(\varphi_{U}\right)=\mathbf{C}$. Consequently, there always exists a faithful normal state $\psi$ on $\mathcal{M}$ such that $\left(\mathcal{M}^{\psi}\right)^{\prime} \cap \mathcal{M}=\mathbf{C}$.

\section{Preliminaries}

2.1. Preliminaries on spectral analysis. We shall need a few definitions and results from the spectral theory of abelian automorphism groups. Let $\left(\alpha_{t}\right)$ be an ultraweakly continuous one-parameter automorphism group on a von Neumann algebra $\mathcal{M}$. For $f \in L^{1}(\mathbf{R})$ and $x \in \mathcal{M}$, set

$$
\alpha_{f}(x)=\int_{-\infty}^{+\infty} f(t) \alpha_{t}(x) d t
$$

The $\alpha$-spectrum $\operatorname{Sp}_{\alpha}(x)$ of $x \in \mathcal{M}$ is defined as the set of characters $\gamma \in \widehat{\mathbf{R}}$ for which $\widehat{f}(\gamma)=0$, for all $f \in L^{1}(\mathbf{R})$ satisfying $\alpha_{f}(x)=0$. We shall identify $\widehat{\mathbf{R}}$ with $\mathbf{R}$ in the usual way such that

$$
\widehat{f}(\gamma)=\int_{-\infty}^{+\infty} e^{i \gamma t} f(t) d t, \quad \forall \gamma \in \mathbf{R}, \forall f \in L^{1}(\mathbf{R}) .
$$

For $z \in \mathbf{C}$, denote by $\Im(z)$ its imaginary part.

Lemma 2.1 ([7]). Let $\mathcal{M}$ and $\left(\alpha_{t}\right)$ be as above. Let $x \in \mathcal{M}$ and $\delta>0$. If the function $t \mapsto \alpha_{t}(x)$ can be extended to an entire (analytic) $\mathcal{M}$-valued function such that

$$
\left\|\alpha_{z}(x)\right\| \leq C e^{\delta|\Im(z)|}, \quad \forall z \in \mathbf{C},
$$

for some constant $C>0$, then $\operatorname{Sp}_{\alpha}(x) \subset[-\delta, \delta]$.

Let $\varphi$ be a f.n. state on a von Neumann algebra $\mathcal{M}$. Denote by $\left(\sigma_{t}^{\varphi}\right)$ the modular group on $\mathcal{M}$ of the state $\varphi$. Denote by $L^{2}(\mathcal{M}, \varphi)$ the $L^{2}$-space associated with $\varphi$ and by $\xi_{\varphi}$ the canonical cyclic separating vector. We shall write $\|x\|_{\varphi}=\varphi\left(x^{*} x\right)^{1 / 2}$, for any $x \in \mathcal{M}$. On bounded subsets of $\mathcal{M}$, the topology given by the norm $\|\cdot\|_{\varphi}$ coincides with the strong operator topology. Recall that $S_{\varphi}^{0}: x \xi_{\varphi} \mapsto x^{*} \xi_{\varphi}$ is a closable (densely defined) operator on $L^{2}(\mathcal{M}, \varphi)$. Denote by $S_{\varphi}$ its closure and 
write $S_{\varphi}=J_{\varphi} \Delta_{\varphi}^{1 / 2}$ for its polar decomposition. Note that $L^{2}(\mathcal{M}, \varphi)$ is naturally endowed with an $\mathcal{M}-\mathcal{M}$ bimodule structure defined as follows:

$$
\begin{aligned}
x \cdot \xi & :=x \xi \\
\xi \cdot x & :=J_{\varphi} x^{*} J_{\varphi} \xi, \quad \forall x \in \mathcal{M}, \forall \xi \in L^{2}(\mathcal{M}, \varphi) .
\end{aligned}
$$

We shall denote $x \cdot \xi$ and $\xi \cdot x$ simply by $x \xi$ and $\xi x$. The next lemma is well-known, but we give a proof for the reader's convenience.

Lemma 2.2. Let $\mathcal{M}$ and $\varphi$ be as above. Let $x \in \mathcal{M}$ and $0<\delta<1$. Assume that $\operatorname{Sp}_{\sigma \varphi}(x) \subset[-\delta, \delta]$. Then $\left\|x \xi_{\varphi}-\xi_{\varphi} x\right\| \leq \delta\|x\|_{\varphi}$.

Proof. Let $x \in \mathcal{M}$ and $0<\delta<1$ be such that $\operatorname{Sp}_{\sigma^{\varphi}}(x) \subset[-\delta, \delta]$. Let $f \in L^{1}(\mathbf{R})$ be such that the Fourier transform $\widehat{f}$ vanishes on $[-\delta, \delta]$. Since $\operatorname{Sp}_{\sigma \varphi}\left(\sigma_{f}^{\varphi}(x)\right) \subset$ $\operatorname{Sp}_{\sigma^{\varphi}}(x) \cap \operatorname{support}(\widehat{f})=\emptyset$ (see [2]), it follows that $\sigma_{f}^{\varphi}(x)=0$. We have

$$
\begin{aligned}
\widehat{f}\left(\log \Delta_{\varphi}\right) x \xi_{\varphi} & =\int_{-\infty}^{+\infty} f(t) \Delta_{\varphi}^{i t} x \xi_{\varphi} d t \\
& =\int_{-\infty}^{+\infty} f(t) \sigma_{t}^{\varphi}(x) \xi_{\varphi} d t \\
& =\sigma_{f}^{\varphi}(x) \xi_{\varphi} \\
& =0 .
\end{aligned}
$$

Thus, by approximating $\mathbf{1}_{\mathbf{R} \backslash[-\delta, \delta]}$ by such functions $\widehat{f}$, we get

$$
\mathbf{1}_{\mathbf{R} \backslash[-\delta, \delta]}\left(\log \Delta_{\varphi}\right) x \xi_{\varphi}=0 ;
$$

i.e. $x \xi_{\varphi}$ is in the spectral subspace of $\log \Delta_{\varphi}$ corresponding to the interval $[-\delta, \delta]$. Notice that

$$
\xi_{\varphi} x=J_{\varphi} x^{*} J_{\varphi} \xi_{\varphi}=J_{\varphi} x^{*} \xi_{\varphi}=J_{\varphi} S_{\varphi} x \xi_{\varphi}=\Delta_{\varphi}^{1 / 2} x \xi_{\varphi} .
$$

Clearly, $\sup \left\{\left|e^{t / 2}-1\right|: t \in[-\delta, \delta]\right\}=e^{\delta / 2}-1$. Moreover, one can see that the operator $\left(1-\Delta_{\varphi}^{1 / 2}\right) \mathbf{1}_{[-\delta, \delta]}\left(\log \Delta_{\varphi}\right)$ is bounded and, to be precise,

$$
\left\|\left(1-\Delta_{\varphi}^{1 / 2}\right) \mathbf{1}_{[-\delta, \delta]}\left(\log \Delta_{\varphi}\right)\right\| \leq e^{\delta / 2}-1 \leq \delta,
$$

since $0<\delta<1$. Thus, we get

$$
\begin{aligned}
\left\|x \xi_{\varphi}-\xi_{\varphi} x\right\| & =\left\|\left(1-\Delta_{\varphi}^{1 / 2}\right) x \xi_{\varphi}\right\| \\
& =\left\|\left(1-\Delta_{\varphi}^{1 / 2}\right) \mathbf{1}_{[-\delta, \delta]}\left(\log \Delta_{\varphi}\right) x \xi_{\varphi}\right\| \\
& \leq\left\|\left(1-\Delta_{\varphi}^{1 / 2}\right) \mathbf{1}_{[-\delta, \delta]}\left(\log \Delta_{\varphi}\right)\right\|\left\|x \xi_{\varphi}\right\| \\
& \leq \delta\|x\|_{\varphi} .
\end{aligned}
$$

Lemma 2.3 ([7]). Let $\mathcal{M}$ and $\varphi$ be as above. Let $\left(x_{n}\right) \in \ell^{\infty}(\mathbf{N}, \mathcal{M})$. Then

$$
\lim _{n}\left\|x_{n} \xi_{\varphi}-\xi_{\varphi} x_{n}\right\|=0 \Longleftrightarrow \lim _{n}\left\|x_{n} \varphi-\varphi x_{n}\right\|=0 .
$$


2.2. Preliminaries on Shlyakhtenko's free Araki-Woods factors. Recall now the construction of the free Araki-Woods factors due to Shlyakhtenko ([14]). Let $H_{\mathbf{R}}$ be a real separable Hilbert space and let $\left(U_{t}\right)$ be an orthogonal representation of $\mathbf{R}$ on $H_{\mathbf{R}}$. Let $H=H_{\mathbf{R}} \otimes_{\mathbf{R}} \mathbf{C}$ be the complexified Hilbert space. Let $J$ be the canonical anti-unitary involution on $H$ defined by

$$
J(\xi+i \eta)=\xi-i \eta, \quad \forall \xi, \eta \in H_{\mathbf{R}}
$$

If $A$ is the infinitesimal generator of $\left(U_{t}\right)$ on $H$, we recall that $j: H_{\mathbf{R}} \rightarrow H$ defined by $j(\zeta)=\left(\frac{2}{A^{-1}+1}\right)^{1 / 2} \zeta$ is an isometric embedding of $H_{\mathbf{R}}$ into $H$. Moreover $J A J=A^{-1}$ and $J A^{i t}=A^{i t} J$, for every $t \in \mathbf{R}$. Let $K_{\mathbf{R}}=j\left(H_{\mathbf{R}}\right)$. It is easy to see that $K_{\mathbf{R}} \cap i K_{\mathbf{R}}=\{0\}$ and that $K_{\mathbf{R}}+i K_{\mathbf{R}}$ is dense in $H$. Write $T=J A^{-1 / 2}$. Then $T$ is an anti-linear closed invertible operator on $H$ satisfying $T=T^{-1}$. Such an operator is called an involution on $H$. Moreover, $K_{\mathbf{R}}=\{\xi \in \operatorname{dom}(T): T \xi=\xi\}$.

We introduce the full Fock space of $H$ :

$$
\mathcal{F}(H)=\mathbf{C} \Omega \oplus \bigoplus_{n=1}^{\infty} H^{\otimes n}
$$

The unit vector $\Omega$ is called the vacuum vector. For any $\xi \in H$, define the left creation operator

$$
\ell(\xi): \mathcal{F}(H) \rightarrow \mathcal{F}(H):\left\{\begin{array}{l}
\ell(\xi) \Omega=\xi \\
\ell(\xi)\left(\xi_{1} \otimes \cdots \otimes \xi_{n}\right)=\xi \otimes \xi_{1} \otimes \cdots \otimes \xi_{n} .
\end{array}\right.
$$

We have $\|\ell(\xi)\|=\|\xi\|$ and that $\ell(\xi)$ is an isometry if $\|\xi\|=1$. For any $\xi \in H$, we denote by $s(\xi)$ the real part of $\ell(\xi)$ given by

$$
s(\xi)=\frac{\ell(\xi)+\ell(\xi)^{*}}{2} .
$$

A crucial result of Voiculescu [16] claims that the distribution of the operator $s(\xi)$ with respect to the vacuum vector state $\varphi(x)=\langle x \Omega, \Omega\rangle$ is the semicircular law of Wigner supported on the interval $[-\|\xi\|,\|\xi\|]$.

Definition 2.4 (Shlyakhtenko, 14]). Let $\left(U_{t}\right)$ be an orthogonal representation of $\mathbf{R}$ on the real Hilbert space $H_{\mathbf{R}}$. The free Araki-Woods von Neumann algebra associated with $\left(H_{\mathbf{R}}, U_{t}\right)$, denoted by $\Gamma\left(H_{\mathbf{R}}, U_{t}\right)^{\prime \prime}$, is defined by

$$
\Gamma\left(H_{\mathbf{R}}, U_{t}\right)^{\prime \prime}:=\left\{s(\xi): \xi \in K_{\mathbf{R}}\right\}^{\prime \prime} .
$$

The vector state $\varphi_{U}(x)=\langle x \Omega, \Omega\rangle$ is called the free quasi-free state and is faithful on $\Gamma\left(H_{\mathbf{R}}, U_{t}\right)^{\prime \prime}$. Let $\xi, \eta \in K_{\mathbf{R}}$ and write $\zeta=\xi+i \eta$. We have

$$
2 s(\xi)+2 i s(\eta)=\ell(\zeta)+\ell(T \zeta)^{*} .
$$

Thus, $\Gamma\left(H_{\mathbf{R}}, U_{t}\right)^{\prime \prime}$ is generated as a von Neumann algebra by the operators of the form $\ell(\zeta)+\ell(T \zeta)^{*}$ where $\zeta \in \operatorname{dom}(T)$. Note that the modular group $\left(\sigma_{t}^{\varphi_{U}}\right)$ of the free quasi-free state $\varphi_{U}$ is given by $\sigma_{-t}^{\varphi_{U}}=\operatorname{Ad}\left(\mathcal{F}\left(U_{t}\right)\right)$, where $\mathcal{F}\left(U_{t}\right)=\operatorname{id} \oplus \bigoplus_{n \geq 1} U_{t}^{\otimes n}$. In particular, it satisfies

$$
\sigma_{-t}^{\varphi_{U}}\left(\ell(\zeta)+\ell(T \zeta)^{*}\right)=\ell\left(U_{t} \zeta\right)+\ell\left(T U_{t} \zeta\right)^{*}, \quad \forall \zeta \in \operatorname{dom}(T), \forall t \in \mathbf{R} .
$$

The free Araki-Woods factors provided many new examples of full factors of type III 1, 2, 11. We can summarize the general properties of the free Araki-Woods factors in the following theorem (see also [15]): 
Theorem 2.5 (Shlyakhtenko, [1, 12, 13, 14]). Let $\left(U_{t}\right)$ be an orthogonal representation of $\mathbf{R}$ on the real Hilbert space $H_{\mathbf{R}}$ with $\operatorname{dim} H_{\mathbf{R}} \geq 2$. Write $\mathcal{M}:=$ $\Gamma\left(H_{\mathbf{R}}, U_{t}\right)^{\prime \prime}$.

(1) $\mathcal{M}$ is a full factor and Connes' invariant $\tau(\mathcal{M})$ is the weakest topology on $\mathbf{R}$ that makes the map $t \mapsto U_{t}$ strongly continuous.

(2) $\mathcal{M}$ is of type $\mathrm{II}_{1}$ iff $U_{t}=\mathrm{id}$, for every $t \in \mathbf{R}$.

(3) $\mathcal{M}$ is of type $\mathrm{III}_{\lambda}(0<\lambda<1)$ iff $\left(U_{t}\right)$ is periodic of period $\frac{2 \pi}{|\log \lambda|}$.

(4) $\mathcal{M}$ is of type $\mathrm{III}_{1}$ in the other cases.

(5) The factor $\mathcal{M}$ has almost periodic states iff $\left(U_{t}\right)$ is almost periodic.

Let $H_{\mathbf{R}}=\mathbf{R}^{2}$ and $0<\lambda<1$. Let

$$
U_{t}^{\lambda}=\left(\begin{array}{cc}
\cos (t \log \lambda) & -\sin (t \log \lambda) \\
\sin (t \log \lambda) & \cos (t \log \lambda)
\end{array}\right)
$$

Notation 2.6 (14]). Write $\left(T_{\lambda}, \varphi_{\lambda}\right):=\left(\Gamma\left(H_{\mathbf{R}}, U_{t}\right)^{\prime \prime}, \varphi_{U}\right)$ where $H_{\mathbf{R}}=\mathbf{R}^{2}$ and $\left(U_{t}\right)$ is given by equation (1).

Using a powerful tool called the matricial model, Shlyakhtenko was able to prove the following isomorphism:

$$
\left(T_{\lambda}, \varphi_{\lambda}\right) \cong\left(\mathbf{B}\left(\ell^{2}(\mathbf{N})\right), \psi_{\lambda}\right) *\left(L^{\infty}[-1,1], \mu\right),
$$

where $\psi_{\lambda}\left(e_{i j}\right)=\delta_{i j} \lambda^{j}(1-\lambda), i, j \in \mathbf{N}$, and $\mu$ is a non-atomic measure on $[-1,1]$. The notation $\cong$ means a state-preserving isomorphism. He also proved that $\left(T_{\lambda}, \varphi_{\lambda}\right)$ has the free absorption property, namely, that

$$
\left(T_{\lambda}, \varphi_{\lambda}\right) * L\left(\mathbf{F}_{\infty}\right) \cong\left(T_{\lambda}, \varphi_{\lambda}\right) .
$$

\section{THE MAIN RESULT}

3.1. Technical lemmas. As we said before, the centralizer of the free quasi-free state may be trivial; this is the case for instance when the orthogonal representation $\left(U_{t}\right)$ on $H_{\mathbf{R}}$ has no eigenvectors. Nevertheless, the following lemma shows that for any free Araki-Woods von Neumann algebra, there exists a non-trivial sequence of unitaries $\left(u_{n}\right)$ in the asymptotic centralizer of the free quasi-free state $\varphi_{U}$.

Lemma 3.1 (Vaes, [15]). Let $\mathcal{M}:=\Gamma\left(H_{\mathbf{R}}, U_{t}\right)^{\prime \prime}$ be a free Araki-Woods von Neumann algebra. Denote by $\varphi$ the free quasi-free state and by $\left(\sigma_{t}\right)$ the modular group of the state $\varphi$. Then there exists a sequence of unitaries $\left(u_{n}\right)$ in $\mathcal{M}$, entire (analytic) w.r.t. $\left(\sigma_{t}\right)$, such that

(1) $\left\|\sigma_{z}\left(u_{n}\right)-u_{n}\right\| \rightarrow 0$ uniformly on compact sets of $\mathbf{C}$,

(2) $\varphi\left(u_{n}\right) \rightarrow 0$,

(3) $\left(u_{n}\right) \in \operatorname{AC}(\varphi)$.

Proof. This lemma, with the exception of item (3), is Vaes' result (see Lemma 4.3 in [15]). Item (3) was not observed by Vaes but is immediate from the construction using Lemmas 2.1, 2.2 and 2.3 .

The following lemma is a generalization of Barnett's lemma (see [1]), which was itself a generalization of Murray and Neumann's $14 \varepsilon$ lemma.

Lemma 3.2 (Vaes, [15]). For $i=1,2$, let $\left(\mathcal{M}_{i}, \varphi_{i}\right)$ be a von Neumann algebra endowed with an f.n. state. Denote by $(\mathcal{M}, \varphi)=\left(\mathcal{M}_{1}, \varphi_{1}\right) *\left(\mathcal{M}_{2}, \varphi_{2}\right)$ the free 
product. Let $a \in \mathcal{M}_{1}$ and $b, c \in \mathcal{M}_{2}$. Assume that $a, b, c$ belong to the domain of $\sigma_{i / 2}^{\varphi}$. Then, for every $x \in \mathcal{M}$,

$$
\|x-\varphi(x) 1\|_{\varphi} \leq \mathcal{E}(a, b, c) \max \left\{\|[x, a]\|_{\varphi},\|[x, b]\|_{\varphi},\|[x, c]\|_{\varphi}\right\}+\mathcal{F}(a, b, c)\|x\|_{\varphi}
$$

where

$$
\begin{aligned}
\mathcal{E}(a, b, c)= & 6\|a\|^{3}+4\|b\|^{3}+4\|c\|^{3}, \\
\mathcal{F}(a, b, c)= & 3 \mathcal{C}(a)+2 \mathcal{C}(b)+2 \mathcal{C}(c)+12\left|\varphi\left(c b^{*}\right)\right|\left\|c b^{*}\right\|, \\
\mathcal{C}(a)= & 2\|a\|^{3}\left\|\sigma_{i / 2}^{\varphi}(a)-a\right\|+2\|a\|^{2}\left\|a^{*} a-1\right\| \\
& +3\left(1+\|a\|^{2}\right)\left\|a a^{*}-1\right\|+6|\varphi(a)|\|a\| .
\end{aligned}
$$

3.2. Proof of the theorem. Let $\mathcal{M}:=\Gamma\left(H_{\mathbf{R}}, U_{t}\right)^{\prime \prime}$ be a free Araki-Woods factor of type $\mathrm{III}_{1}$ and denote by $\varphi$ the free quasi-free state. We recall that such a factor can always be written as the free product of three free Araki-Woods von Neumann algebras (see the proof of Theorem 2.7 in [15]):

$$
(\mathcal{M}, \varphi) \cong\left(\mathcal{M}_{1}, \varphi_{1}\right) *\left(\mathcal{M}_{2}, \varphi_{2}\right) *\left(\mathcal{M}_{3}, \varphi_{3}\right) .
$$

Notice that $\sigma_{t}^{\varphi}=\sigma_{t}^{\varphi_{1}} * \sigma_{t}^{\varphi_{2}} * \sigma_{t}^{\varphi_{3}}, \forall t \in \mathbf{R}$.

Thanks to Lemma 3.1, we may choose three sequences of unitaries $\left(u_{n}^{j}\right)$, for $j \in\{1,2,3\}$, such that $u_{n}^{j} \in \mathcal{U}\left(\mathcal{M}_{j}\right)$ is analytic w.r.t. $\left(\sigma_{t}^{\varphi_{j}}\right)$ and satisfies conditions (1) - (3) of Lemma 3.1, for all $j \in\{1,2,3\}$. The way the sequence of unitaries $\left(u_{n}^{j}\right)$ is constructed in Lemma 3.1 (see Lemma 4.3 in [15]) shows that conditions (1) - (3) are satisfied for the state $\varphi$; i.e. the sequence of unitaries $\left(u_{n}^{j}\right)$ in $\mathcal{M}_{j}$ satisfies, for every $j \in\{1,2,3\}$,

(1) $\left\|\sigma_{z}^{\varphi}\left(u_{n}^{j}\right)-u_{n}^{j}\right\| \rightarrow 0$ uniformly on compact sets of $\mathbf{C}$,

(2) $\varphi\left(u_{n}^{j}\right) \rightarrow 0$

(3) $\left\|\left[u_{n}^{j}, \varphi\right]\right\| \rightarrow 0$.

Moreover, by freeness, $\varphi\left(u_{n}^{3}\left(u_{n}^{2}\right)^{*}\right)=\varphi\left(u_{n}^{3}\right) \overline{\varphi\left(u_{n}^{2}\right)} \rightarrow 0$.

Assume that $a \in \operatorname{AB}(\varphi)$. Fix $\varepsilon>0$. Since $\left(u_{n}^{j}\right) \in \mathrm{AC}(\varphi)$, it follows that $\left[a, u_{n}^{j}\right] \rightarrow 0$ ultrastrongly for any $j \in\{1,2,3\}$, and thus we may choose $n \in \mathbf{N}$ large enough such that

$$
\begin{aligned}
\left\|\left[a, u_{n}^{j}\right]\right\|_{\varphi} & \leq \varepsilon / 28, \quad \forall j \in\{1,2,3\}, \\
\mathcal{F}\left(u_{n}^{1}, u_{n}^{2}, u_{n}^{3}\right)\|a\|_{\varphi} & \leq \varepsilon / 2 .
\end{aligned}
$$

Thus, thanks to Lemma 3.2, we get $\|a-\varphi(a) 1\|_{\varphi} \leq \varepsilon / 2+\varepsilon / 2=\varepsilon$. Since $\varepsilon>0$ is arbitrary, $a=\varphi(a) 1$. Thus $\mathrm{AB}(\varphi)=\mathbf{C}$, and we are done.

3.3. Final remark. Set $\mathcal{M}:=\Gamma\left(L^{2}(\mathbf{R}, \mathbf{R}), \lambda_{t}\right)^{\prime \prime}$, the free Araki-Woods factor associated with the left regular representation $\left(\lambda_{t}\right)$ of $\mathbf{R}$ on the real Hilbert space $L^{2}(\mathbf{R}, \mathbf{R})$. Shlyakhtenko showed in $[13$ that the continuous core of $\mathcal{M}$ is isomorphic to $L\left(\mathbf{F}_{\infty}\right) \otimes \mathbf{B}\left(\ell^{2}\right)$ and that the dual action is precisely the one constructed by Rădulescu in [9]. As observed in [10, for any f.n. state $\varphi$ on $\mathcal{M}$, the centralizer $\mathcal{M}^{\varphi}$ is amenable. Indeed, first we have

$$
\mathcal{M} \rtimes_{\sigma^{\varphi}} \mathbf{R} \simeq L\left(\mathbf{F}_{\infty}\right) \otimes \mathbf{B}\left(\ell^{2}\right) .
$$

Choose on the left-hand side of (21) a non-zero projection $p \in L(\mathbf{R})$ such that $\operatorname{Tr}(p)<+\infty$. We know that $p\left(\mathcal{M} \rtimes_{\sigma \varphi} \mathbf{R}\right) p \simeq L\left(\mathbf{F}_{\infty}\right)$ is solid by Ozawa's result (무). Since $L(\mathbf{R}) p$ is diffuse in $p\left(\mathcal{M} \rtimes_{\sigma^{\varphi}} \mathbf{R}\right) p$, its relative commutant must be amenable. 
In particular $\mathcal{M}^{\varphi} \otimes L(\mathbf{R}) p$ is amenable. Thus, $\mathcal{M}^{\varphi}$ is amenable. Consequently, we obtain

Corollary 3.3. Let $\mathcal{M}:=\Gamma\left(L^{2}(\mathbf{R}, \mathbf{R}), \lambda_{t}\right)^{\prime \prime}$. Then there exists an f.n. state $\psi$ on $\mathcal{M}$ such that $\left(\mathcal{M}^{\psi}\right)^{\prime} \cap \mathcal{M}=\mathbf{C}$. Moreover, $\mathcal{M}^{\psi}$ is isomorphic to the unique hyperfinite $\mathrm{II}_{1}$ factor.

\section{ACKNOWLEDGEMENTS}

Part of this work was done while the author was visiting the University of Tokyo. He gratefully thanks Professors Y. Kawahigashi and N. Ozawa for their kind invitation.

\section{REFERENCES}

[1] L. Barnett, Free product von Neumann algebras of type III. Proc. Amer. Math. Soc. 123 (1995), 543-553. MR1224611 (95c:46096)

[2] A. Connes, Une classification des facteurs de type III. Ann. Sci. École Norm. Sup. 6 (1973), 133-252. MR0341115 (49:5865)

[3] A. Connes, Almost periodic states and factors of type $\mathrm{III}_{1}$. J. Funct. Anal. 16 (1974), 415-445. MR0358374 (50:10840)

[4] A. Connes, Factors of type $\mathrm{III}_{1}$, property $L_{\lambda}^{\prime}$ and closure of inner automorphisms. J. Operator Theory 14 (1985), 189-211. MR789385 (88b:46088)

[5] A. Connes and E. Størmer, Homogeneity of the state space of factors of type $\mathrm{III}_{1}$. J. Funct. Anal. 28 (1978), 187-196. MR0470689 (57:10435)

[6] K. Dykema, Free products of finite-dimensional and other von Neumann algebras with respect to non-tracial states. Free probability theory (Waterloo, ON, 1995), Fields Inst. Commun. 12. Amer. Math. Soc., Providence, RI, 1997, pp. 41-88. MR.1426835 (98c:46131)

[7] U. Haagerup, Connes' bicentralizer problem and uniqueness of the injective factor of type III $_{1}$. Acta Math. 69 (1986), 95-148. MR880070 (88f:46117)

[8] N. Ozawa, Solid von Neumann algebras. Acta Math. 192 (2004), 111-117. MR 2079600 (2005e:46115)

[9] F. Rădulescu, A one-parameter group of automorphisms of $L\left(\mathbf{F}_{\infty}\right) \otimes \mathbf{B}(H)$ scaling the trace. C. R. Acad. Sci. Paris Sér. I Math. 314 (1992), 1027-1032. MR1168529 (93i:46111)

[10] D. Shlyakhtenko, On multiplicity and free absorption for free Araki-Woods factors. arXiv:math.OA/0302217

[11] D. Shlyakhtenko, On the classification of full factors of type III. Trans. Amer. Math. Soc. 356 (2004), 4143-4159. MR2058841 (2005a:46124)

[12] D. Shlyakhtenko, A-valued semicircular systems. J. Funct. Anal. 166 (1999), 1-47. MR:1704661 (2000j:46124)

[13] D. Shlyakhtenko, Some applications of freeness with amalgamation. J. Reine Angew. Math. 500 (1998), 191-212. MR1637501 (99j:46079)

[14] D. Shlyakhtenko, Free quasi-free states. Pacific J. Math. 177 (1997), 329-368. MR1444786 (98b:46086)

[15] S. Vaes, États quasi-libres libres et facteurs de type III (d'après D. Shlyakhtenko). Séminaire Bourbaki, exposé 937, Astérisque 299 (2005), 329-350. MR 2167212 (2007d:46056)

[16] D.-V. Voiculescu, K.J. Dykema and A. Nica, Free random variables. CRM Monograph Series 1. Amer. Math. Soc., Providence, RI, 1992. MR1217253 (94c:46133)

Department of Mathematics, University of California, Los Angeles, 520 Portola Plaza, Los Angeles, California 90095

E-mail address: cyril@math.ucla.edu

Current address: CNRS-ENS Lyon, UMPA UMR 5669, 69364 Lyon cedex 7, France

E-mail address: cyril.houdayer@umpa.ens-lyon.fr 